\title{
METABOLIC EFFECTS OF MARKED SODIUM RESTRICTION IN HYPERTENSIVE PATIENTS: CHANGES IN TOTAL EX- CHANGEABLE SODIUM AND POTASSIUM ${ }^{1}$
}

\author{
BY LEWIS K. DAHL, BERNARD G. STALL, III, AND GEORGE C. COTZIAS
}

(From the Department of Medicine, Brookhaven National Laboratory, Upton, New York)

(Submitted for publication February 16, 1954; accepted June 16, 1954)

It is important to understand the mechanism by which drastic sodium restriction effects a decrease in blood pressure in some patients with essential hypertension. It has been shown (1) that a decline in total exchangeable sodium (TENa) as measured with the isotope $\mathrm{Na}^{24}$, is not correlated with a decline in blood pressure, and therefore simple "desalting" of the body can not account for the phenomenon. The important relationship which exists between sodium and potassium in most living species, both animal and plant, suggested the possibility that sodium restriction might be effective in lowering blood pressure through secondary effects on $\mathrm{K}$. With the isotopes $\mathrm{Na}^{24}$ and $\mathrm{K}^{42}$, nearly all of the body's $\mathrm{Na}$ and $\mathrm{K}$ pools can be measured $(2,3)$. Therefore, it is possible to quantitate accurately the effects of marked $\mathrm{Na}$ restriction upon these pools and to determine whether the effects of $\mathrm{Na}$ restriction on total exchangeable potassium (TEK) may be correlated with alterations in blood pressure. The present paper is a report of a study on 11 patients with essential hypertension who were given a basic low $\mathrm{Na}$ diet supplemented during the period of control observations with 10 grams ( $180 \mathrm{mEq}$.) of enteric coated $\mathrm{NaCl}$ per day. The removal of this added $\mathrm{NaCl}$ during the subsequent test period constituted the sole change in regimen. During both phases of the study after suitable periods for equilibration the effects on TENa, TEK, and blood pressure were studied.

\section{PROCEDURE}

\section{Patients and clinical status}

Eleven adult patients with essential hypertension were studied for a 3-month period on the metabolic wards of the Brookhaven National Laboratory Hospital. These people were admitted in two groups; one consisted of two males and four females from April to July (Group I), the

1 This work was carried out under the auspices of the Atomic Energy Commission. other of three males and two females from September to December (Group II), 1953. Since the primary purpose of this communication is not concerned with the clinical effects of sodium restriction which have been reported frequently in the past (4-6) itemized details of each patient's status relative to laboratory findings have been omitted from this report except as seemed pertinent. With the two exceptions noted below (patients $M$ and $R$ ) all patients were of the type classed as benign essential hypertension. Urea clearances were normal in all, but variable degrees of albuminuria were present in some patients. Intravenous pyelograms were normal except in patient $\mathrm{R}$ as noted. Serum cholesterol, $\mathrm{CO}_{2}, \mathrm{Cl}$, total protein, albumin and globulin, sodium and potassium were within normal limits in all patients. Electrocardiograms ranged from normal to variable degrees of left axis deviation and low or inverted $T$ waves. (Tracings on patient $\mathbf{R}$ revealed classical evidence of acute myocardial infarction involving the anterior wall of the left ventricle during the two episodes described subsequently.) Heart size was shown by X-ray to vary from normal in some to moderate, diffuse enlargement in others. None gave past histories which suggested episodes of acute heart failure. Benzodioxane tests for pheochromocytoma were negative in all. Both males mentioned earlier had serious disease; $M$, age 53 , had had a right hemiplegia six months prior to admission with residual weakness and spasticity. Upon admission, he was found to have mild diabetes mellitus as well, which was readily controlled by small doses of protamine zinc insulin. During his hospital stay, he had two transient cerebrovascular thromboses, from both of which return to previous level of function occurred in three to seven days. This patient's disease was obviously severe and about six weeks after his initial discharge he died from multiple cerebrovascular thromboses. Patient $R$, age 47 , who had had angina on effort for several years, developed two mild coronary occlusions five and seven weeks after admission with uneventful recovery. He had had a chronic duodenal ulcer of more than 20 years duration which plagued both patient and staff during the study. By intravenous pyelogram he was shown to have a small asymptomatic calculus in each kidney, measuring 5 and $15 \mathrm{~mm}$. in diameter, respectively; these were presumed to be secondary to the heavy alkali and milk ingestion for many years.

\section{Experimental program}

The patients remained ambulatory throughout their stay except during studies or intercurrent illnesses. Upon 
admission, each patient was placed on a basic diet calculated to yield about $6 \mathrm{mEq}$. sodium per day (analysis of 18 separate diets yielded a mean value of $5.7 \mathrm{mEq}$. with S.D. \pm 1.4 ) ; because of this restriction the daily protein intake averaged approximately 55 grams. In Group I, protein supplement, although allowed, was not required to be eaten; associated with this, members of the group lost variable and often significant amounts of weight. Since previous work (7) had shown that the levels of protein in the diet had no effect upon blood pressure, weight loss seemed unimportant. In view of the changes in TENa and TEK, subsequently calculated to be present, it was thought necessary in the future to control the factor of weight loss as well. Therefore, members of the second group were required to consume a total of 1.5 grams of protein per $\mathrm{Kg}$. per day through low $\mathrm{Na}$ protein supplements with Lesofac ${ }^{2}$ or Lonalac ${ }^{8}$; on this regimen, the weights remained within narrow limits, with the maximal change recorded being a gain of $4.0 \mathrm{Kg}$. in patient B. Salt-free or salt-poor carbohydrates and fats were given in amounts sufficient to satisfy caloric needs. The diets were calculated to contain 30 to $100 \mathrm{mEq}$. of potassium per day and this was confirmed by analyses. The first five to six weeks served as the control period; therefore, during these first weeks 10 grams (180 mEq.) of sodium chloride in enteric coated capsules were administered daily to each patient in divided doses, an amount which is regarded as being within the average of American intakes (8). At the termination of the control observations, the sole change in the regimen was the omission of the added $\mathrm{NaCl}$, so that the effects of this restriction alone could be studied. At intervals before and after this sharp decrease in $\mathrm{NaCl}$ intake, TENa and TEK were measured as well as other studies appropriate for evaluation of clinical status.

\section{METHODS}

Blood pressure, urine and blood collections; sodium and potassium analyses

Blood pressures were measured six mornings a week by one of the authors under standard conditions; namely, after breakfast with a minimum bed rest of half an hour before the readings were made. Systolic and diastolic pressures were recorded as the first appearance and total disappearance of sound respectively as recommended by a committee of the American Heart Association (9). All urines in each 24-hour period were pooled, the volumes measured, aliquots removed, and then preserved with 1 per cent $\mathrm{HCl}$ for subsequent chemical analysis of sodium and potassium with a Perkin-Elmer Model 52A flame photometer, with a lithium internal standard. The volume of the urine samples used for analysis ranged from 1 to $40 \mathrm{ml}$. depending upon the concentration of $\mathrm{Na}$ or $\mathrm{K}$ in the specimen. Blood samples were removed without stasis, mixed in heparin tubes and centrifuged within 5 to 10 minutes. With care in handling the samples visible

2 Obtained through the courtesy of Wyeth Laboratories, Philadelphia, Pennsylvania. hemolysis was not seen. From the plasma so obtained, $1 \mathrm{ml}$. samples were analyzed in duplicate with the flame photometer in the fashion used for urine.

\section{Total exchangeable sodium (TENa)}

TENa was determined by an isotope dilution technique with the procedure essentially as described earlier (1). The isotope $\mathrm{Na}^{24} \mathrm{CO}_{3}$ was obtained from the Brookhaven National Laboratory reactor, sterilized, then prepared for administration by diluting with 5 per cent dextrose; of this each patient received about $100 \mu \mathrm{c}$ (containing approximately $.06 \mathrm{mEq} . \mathrm{Na}^{23}$ ) intravenously from a calibrated $100 \mathrm{ml}$. buret over a 7 - to 10 -minute period. Plasma samples were always taken at 5, 7, and 24 hours in the first group and at 24 and 26 hours in the second group; the final 24- and 26-hour specimens were always collected with the patients in the fasting state. Thus, in Group I the value for final exchangeable sodium represented the corrected 24-hour figure and was in all instances a reasonable extension of the values found earlier. In Group II, the final figure was the mean of the 24- and 26-hour values at both of which times presumably the maximal value for $\mathrm{TENa}$ had been reached. Based on the data of Edelman, James, Baden, and Moore (2) it is highly improbable that a much longer period of equilibration would have affected the values for TENa obtained here. During the low sodium phase of these and related studies, correction for $\mathrm{Na}^{*}$ excretion in the urine was found to be negligible whereas during the high sodium phase the average 24-hour excretion was 6.3 per cent (range 3.2 to 10.0 per cent) of the injected dose.

Radioactivity was measured with a Texaco well-type Geiger counter and standard scaler; $5 \mathrm{ml}$. liquid samples were counted until at least 10,000 counts above background were recorded in duplicate on specimens of blood, urine, and standards. During the low sodium phase, however, urines were counted only long enough to demonstrate that the specific activity was not significantly above background. Correction for decay was made by counting at precisely spaced intervals seriatim Standard No. 1, Sample No. 1, Sample No. 2, Standard No. 2 with the counting intervals so arranged that the average of the times of counting samples and standards were at the same mid-point in time, with decay corrections having been made automatically thereby.

\section{Total exchangeable potassium (TEK)}

TEK was measured by an isotope dilution technique (3). Approximately 100 to $150 \mu \mathrm{c} \mathrm{K} \mathrm{K}^{42} \mathrm{CO}_{3}$ obtained from the Brookhaven reactor (containing about $.04 \mathrm{mEq} . \mathrm{K}^{20}$ ) were administered by the same technique used for estimation of exchangeable sodium. In Group I, all urines were pooled up to 37 hours with spot specimens collected after an overnight fast at 38,40 , and 42 hours, at which times probably 95 per cent of the total body potassium is in equilibration with $\mathrm{K}^{22}(3)$. The average specific activity of these three samples, corrected for loss of the isotope in urine, was used to calculate total exchangeable potassium. The average excretion of the administered $\mathrm{K}^{42}$ at 40 hours in 34 consecutive determinations was 5.5 
per cent (range 1.2 to 8.7 per cent) and did not change during either phase of the present study. In Group II, the period allowed for equilibration was an average of 20 hours with all urine pooled up to 17 hours and spot samples collected under fasting conditions at 18,20 , and 22 hours. In no instance did it appear that equilibration had failed to occur in these times as manifested by a regular increase in TEK. If these 20 -hour determinations underestimated the exchangeable $\mathrm{K}$ slightly relative to the 40-hour values (3), since each patient was his own control, the final relative values during high and low $\mathrm{Na}$ would not be affected. A cation exchange resin of the sulfonic type (Amberlite IR-120) was used to concentrate the urine potassium and thereby enhance the counting rate approximately eight-fold. Counting was done with a Harshaw scintillation crystal using a special input amplifier designed at the Brookhaven Laboratory and an Atomic Scaler (Model 1050A). Three ml. liquid samples of standard and sample were counted in the fashion described for $\mathrm{Na}^{24}$ using the same technique for decay correction.

\section{Statistical analyses of significance}

The total exchangeable sodium values were calculated from the following data: serum sodium concentration, the volume and counting rate of the isotopic solution administered with correction for the amount excreted where this was significant, and the counting rate of serum at the time of equilibration. Serum sodium determinations in duplicate were reproducible within $0.5 \mathrm{mEq}$., which amounts to less than a 0.4 per cent difference. Recovery experiments in which standard saline solutions were added to plasma gave maximal errors of 1 per cent; therefore, an error of 1 per cent has been allowed. The infusion fluid was administered in a $100 \mathrm{ml}$. buret, calibrated to contain $100.14 \mathrm{ml}$; this correction has been disregarded. In this series, the average counting rate of infusion fluid and serum were 5000 and 2500 counts per minute (cpm), with total counts on duplicate samples averaging 27,500 , and 25,000 , respectively. The coefficient of variation calculated from standard deviation is respectively, .0063 and .0060 (10). Because of local conditions (both a reactor and 2.5 billion electron volt proton accelerator within 1.5 miles) background averaged 400 cpm, with a total of about 70,000 background counts per study. The coefficient of variation here amounts to .0040 and has been included in the calculation of total error. The final cumulative error has been calculated from the formula $\sigma^{2}=\sqrt{\sigma_{1}^{2}+\sigma_{2}^{2}} \ldots$ (11) where $\sigma$ equals cumula-

\begin{tabular}{|c|c|c|c|c|c|c|c|c|c|c|c|c|c|c|c|c|c|c|}
\hline \multirow[b]{4}{*}{ Pt. } & \multicolumn{18}{|c|}{ Dets on Patients vith Significant Doclines in both S } \\
\hline & \multirow{2}{*}{\multicolumn{4}{|c|}{$\begin{array}{l}\text { Blood Pressure } \\
\text { (Moen \& S.D.) }\end{array}$}} & \multirow{3}{*}{$\begin{array}{l}\text { Deys } \\
\text { before }(-) \text { or } \\
\text { after }(+) \text { wa } \\
\text { Restriction }\end{array}$} & \multirow{2}{*}{\multicolumn{3}{|c|}{$\begin{array}{l}\text { Body WeIght } \\
\text { (IB) }\end{array}$}} & \multicolumn{5}{|c|}{ Exchangeable spolive Data } & \multicolumn{5}{|c|}{ Inchangoable Potassilu Dats } \\
\hline & & & & & & & & & \multirow[b]{2}{*}{$\begin{array}{l}\text { Total } \\
\text { (moq) }\end{array}$} & \multirow[b]{2}{*}{\begin{tabular}{|c|}
$\begin{array}{c}\text { Por K8 } \\
\text { (meg/L8) }\end{array}$ \\
\end{tabular}} & \multicolumn{3}{|c|}{\begin{tabular}{l|} 
Difference from \\
Proceding studf $x$
\end{tabular}} & \multirow[b]{2}{*}{$\begin{array}{l}\text { Total } \\
(\text { meq) }\end{array}$} & \multirow[b]{2}{*}{ 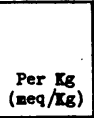 } & \multicolumn{3}{|c|}{$\begin{array}{l}\text { Difference from } \\
\text { Proceding study } \times\end{array}$} \\
\hline & syst. & $p$ & Diagt. & $p$ & & Admission & study & $\underset{\text { Admission }}{\Delta}$ & & & $\underset{\text { Days }}{\Delta}$ & $\Delta w_{(\mathrm{kg})}^{\Delta \mathrm{t}_{j}}$ & $\begin{array}{r}\Delta \text { IEsta } \\
\text { (meq) }\end{array}$ & & & $\Delta$ & $\begin{array}{c}\Delta \mathbf{w t}_{\mathrm{t}} \\
\left(\mathrm{K}_{\mathrm{B}}\right)\end{array}$ & $\begin{array}{l}\Delta \max \\
(\operatorname{mog})\end{array}$ \\
\hline 1 & 2 & 3 & 4 & 5 & 6 & 7 & 8 & 9 & 10 & 31 & 12. & 13 & 24 & 15 & 16 & 17 & 18 & 19 \\
\hline \multirow[t]{2}{*}{$\left(\begin{array}{c}c \\
39 \\
(3+20)\end{array}\right.$} & $\begin{array}{l}162 \\
(10) \\
\end{array}$ & & 1111 & & $\begin{array}{l}-35 \\
-28 \\
-21 \\
-12 \\
-8 \\
\end{array}$ & 71.7 & $\begin{array}{l}70.5 \\
70.4 \\
69.7 \\
69.7 \\
\end{array}$ & $\begin{array}{l}-1.2 \\
-1.3 \\
-2.0 \\
-2.0 \\
\end{array}$ & 2531 & 35.9 & 20 & -0.8 & -88 & $\begin{array}{l}2558 \\
2391\end{array}$ & $\begin{array}{l}36.3 \\
34.3\end{array}$ & 9 & -0.7 & -167 \\
\hline & $\begin{array}{l}130 \\
(6)\end{array}$ & $<.01$ & $\begin{array}{l}94 \\
\text { (6) }\end{array}$ & $<.01$ & $\begin{array}{r}+19 \\
+27 \\
+39 \\
+47 \\
+51 \\
\end{array}$ & & $\begin{array}{l}69.0 \\
68.7 \\
68.6 \\
68.2 \\
68.0\end{array}$ & $\begin{array}{l}-2.7 \\
-3.0 \\
-3.1 \\
-3.5 \\
-3.7\end{array}$ & 2320 & $\begin{array}{l}33.8 \\
34.7\end{array}$ & $\begin{array}{l}35 \\
24\end{array}$ & $\begin{array}{c}-1.0 \\
-0.7 \\
\end{array}$ & $\begin{array}{r}-123 \\
+39 \\
\end{array}$ & $\begin{array}{l}2253 \\
2284 \\
2320\end{array}$ & $\begin{array}{l}.32 .7 \\
33.3 \\
34.0\end{array}$ & $\begin{array}{l}31 \\
20 \\
8\end{array}$ & $\begin{array}{c}-0.7 \\
-0.4 \\
-0.4\end{array}$ & $\begin{array}{l}-238 \\
+29 \\
+36\end{array}$ \\
\hline \multirow[t]{2}{*}{$\begin{array}{l}\hat{8}+42 \\
(45455)\end{array}$} & $\begin{array}{l}139 \\
\text { (9) }\end{array}$ & & $\begin{array}{l}97 \\
\text { (6) }\end{array}$ & & $\begin{array}{l}-42 \\
-19 \\
-16 \\
-12 \\
-9\end{array}$ & 82.4 & $\begin{array}{l}81.4 \\
8.2 \\
81.4 \\
81.9\end{array}$ & $\begin{array}{l}-1.0 \\
-0.2 \\
-1.0 \\
-0.5\end{array}$ & $\begin{array}{l}3127 \\
3207\end{array}$ & $\begin{array}{l}38.4 \\
39.1\end{array}$ & 3 & +0.8 & +80 & $\begin{array}{l}2879 \\
2935\end{array}$ & $\begin{array}{l}35.4 \\
35.8\end{array}$ & 3 & +0.5 & +36 \\
\hline & $\begin{array}{l}133 \\
(14) \\
\end{array}$ & $<.01$ & $\begin{array}{l}83 \\
\text { (7) }\end{array}$ & $<.02$ & $\begin{array}{r}+30 \\
+37 \\
+40 \\
+46 \\
+46 \\
+53\end{array}$ & & $\begin{array}{l}81.0 \\
81.3 \\
81.5 \\
81.9 \\
81.5 \\
81.5 \\
\end{array}$ & $\begin{array}{l}-2.4 \\
-1.1 \\
-0.9 \\
-0.5 \\
-0.9 \\
-0.9 \\
\end{array}$ & $\begin{array}{l}2922 \\
2922 . \\
2968\end{array}$ & $\begin{array}{l}36.1 \\
36.1 \\
36.5\end{array}$ & $\begin{array}{l}46 \\
10 \\
6\end{array}$ & $\begin{array}{r}-1.2 \\
+0.5 \\
0.0\end{array}$ & $\begin{array}{c}-285 \\
0 \\
+46\end{array}$ & $\begin{array}{l}3248 \\
3231 \\
3021 \\
\end{array}$ & $\begin{array}{l}40.0 \\
39.5 \\
37.1 \\
\end{array}$ & $\begin{array}{l}46 \\
7 \\
9 \\
\end{array}$ & $\begin{array}{l}-0.6 \\
+0.6 \\
-0.4 \\
\end{array}$ & $\begin{array}{l}+313 \\
-17 \\
-201 \\
\end{array}$ \\
\hline \multirow[t]{2}{*}{$\begin{array}{c}\operatorname{son}_{45} \\
(54 h 72)\end{array}$} & $\begin{array}{l}218 \\
(14)\end{array}$ & & $\begin{array}{l}135 \\
\text { (8) }\end{array}$ & & $\begin{array}{l}-12 \\
-19 \\
-16 \\
-12 \\
-9\end{array}$ & 83.1 & $\begin{array}{l}82.1 \\
82.2 \\
88.7 \\
81.9 \\
\end{array}$ & $\begin{array}{l}-1.0 \\
-0.9 \\
-1.4 \\
-1.2 \\
\end{array}$ & 3190 & $\begin{array}{l}38.9 \\
37.4\end{array}$ & 3 & +0.1 & -115 & $\begin{array}{l}2844 \\
2860 \\
\end{array}$ & $\begin{array}{r}34.8 \\
34.9 \\
\end{array}$ & 3 & +0.2 & +16 \\
\hline & $\begin{array}{l}199 \\
(15)\end{array}$ & $<.01$ & $\begin{array}{l}124 \\
(8)\end{array}$ & $<.01$ & $\begin{array}{r}+30 \\
+37 \\
+40 \\
+44 \\
+46 \\
+53 \\
\end{array}$ & & $\begin{array}{l}82.5 \\
83.3 \\
83.3 \\
83.5 \\
83.4 \\
83.9 \\
\end{array}$ & $\begin{array}{l}+0.6 \\
+0.2 \\
+0.2 \\
+0.4 \\
+0.3 \\
+0.8 \\
\end{array}$ & $\begin{array}{l}2974 \\
3057 \\
2991\end{array}$ & $\begin{array}{l}36.0 \\
36.7 \\
35.9\end{array}$ & $\begin{array}{l}46 \\
10 \\
6\end{array}$ & $\begin{array}{l}+0.3 \\
+0.8 \\
+0.1\end{array}$ & $\begin{array}{l}-101 \\
+83 \\
-66\end{array}$ & $\begin{array}{l}3329 \\
3223 \\
2962 \\
\end{array}$ & $\begin{array}{l}40.0 \\
38.6 \\
35.3 \\
\end{array}$ & $\begin{array}{l}46 \\
7 \\
9\end{array}$ & $\begin{array}{l}+1.4 \\
+0.2 \\
+0.4\end{array}$ & $\begin{array}{l}+469 \\
-106 \\
-261 \\
\end{array}$ \\
\hline \multicolumn{19}{|c|}{ 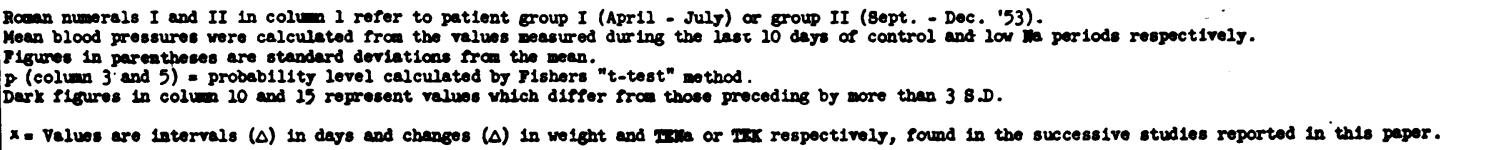 } \\
\hline
\end{tabular}




\begin{tabular}{|c|c|c|c|c|c|c|c|c|c|c|c|c|c|c|c|c|c|c|}
\hline \multirow[b]{4}{*}{ Pt. } & \multicolumn{18}{|c|}{$\begin{array}{l}\text { TNBUS Th } \\
\text { Data on Patients with Significant Decline in Only Systolic or Dlastolic Pressures }\end{array}$} \\
\hline & \multirow{2}{*}{\multicolumn{4}{|c|}{$\begin{array}{l}\text { Blood Pressure } \\
\text { (Mean \& S.D.) }\end{array}$}} & \multirow{3}{*}{$\begin{array}{c}\text { Deys } \\
\text { before ( (-) or } \\
\text { after (+) wa } \\
\text { Restriction }\end{array}$} & \multirow{2}{*}{\multicolumn{3}{|c|}{$\begin{array}{l}\text { Body We Lght } \\
\text { (KB) }\end{array}$}} & \multicolumn{5}{|c|}{ Exchangeable sodiva Dats } & \multicolumn{5}{|c|}{ Ixchangeable Potassive Date } \\
\hline & & & & & & & & & \multirow[b]{2}{*}{$\begin{array}{l}\text { Total } \\
\text { (moq) }\end{array}$} & \multirow[b]{2}{*}{$\begin{array}{c}\text { Per } \mathbf{~ r g} \\
(\mathbf{m o q} / \mathbf{R g})\end{array}$} & \multicolumn{3}{|c|}{$\begin{array}{l}\text { Difference Pron } \\
\text { Preceding Study } x\end{array}$} & \multirow[b]{2}{*}{$\begin{array}{l}\text { Total } \\
(\text { (o) }) \\
\end{array}$} & \multirow[b]{2}{*}{$\begin{array}{l}\text { Por } \mathrm{K}_{8} \\
\left(\mathbf{m e q} / \mathrm{K}_{\mathrm{g}}\right)\end{array}$} & \multicolumn{3}{|c|}{$\begin{array}{l}\text { Difforonce froca } \\
\text { Proceding study } x\end{array}$} \\
\hline & syst. & p & Diast. & p & & Admission & study & $\underset{\text { Aaniseion }}{\Delta}$ & & & $\Delta$ & $\Delta \mathrm{wt}_{(\mathrm{kB})}$ & $\underset{(\operatorname{meq})}{\Delta \operatorname{Tan}}$ & & & $\Delta$ & \begin{tabular}{|l|}
$\Delta w_{t}$ \\
$\left(\mathbf{r t g}_{\mathbf{g}}\right)$
\end{tabular} & $\begin{array}{l}\Delta \max \\
(\operatorname{mog})\end{array}$ \\
\hline 1 & 2 & 3 & 4 & 5 & 6 & 7 & 8 & 9 & 10 & 11 & 12 & 13 & 14 & 15 & 16 & 17 & 18 & 19 \\
\hline \multirow[t]{2}{*}{$\underset{85274)}{8}$} & $\begin{array}{l}285 \\
\text { (10) }\end{array}$ & & $\begin{array}{l}104 \\
(7)\end{array}$ & & $\begin{array}{l}-35 \\
-28 \\
-21 \\
-12 \\
-8\end{array}$ & 56.8 & $\begin{array}{l}56.3 \\
55.8 \\
55.2 \\
55.0\end{array}$ & $\begin{array}{l}-0.5 \\
-1.0 \\
-1.6 \\
-1.8\end{array}$ & $\begin{array}{c}1900 \\
\cdot \\
1925\end{array}$ & $\begin{array}{l}33.7 \\
35.0\end{array}$ & 20 & -1.3 & +25 & $\begin{array}{l}1867 \\
1983\end{array}$ & $\begin{array}{l}33.5 \\
35.9\end{array}$ & 9 & -0.6 & +116 \\
\hline & $\begin{array}{l}157 \\
\text { (10) }\end{array}$ & $<.01$ & $\begin{array}{l}98 \\
(8)\end{array}$ & $<.2$ & $\begin{array}{r}+19 \\
+27 \\
+39 \\
+47 \\
+51\end{array}$ & & $\begin{array}{l}53.7 \\
53.0 \\
53.2 \\
52.4 \\
52.7\end{array}$ & $\begin{array}{r}-3.1 \\
-3.8 \\
-3.6 \\
4.4 \\
-4.1\end{array}$ & $\begin{array}{l}1747 \\
1801\end{array}$ & $\begin{array}{l}33.0 \\
34.2\end{array}$ & 35. & $\begin{array}{l}-2.0 \\
-0.3\end{array}$ & $\begin{array}{r}-178 \\
+54 \\
\end{array}$ & $\begin{array}{l}1926 \\
1807 \\
1793\end{array}$ & $\begin{array}{l}35.9 \\
34.0 \\
34.2\end{array}$ & $\begin{array}{l}31 \\
20 \\
8\end{array}$ & $\begin{array}{l}-15 \\
-0.5 \\
-0.8\end{array}$ & $\mid \begin{array}{l}+57 \\
-119 \\
-14\end{array}$ \\
\hline \multirow{2}{*}{$\begin{array}{c}P \\
\$ 72 \\
(\$ 5424)\end{array}$} & $\begin{array}{l}182 \\
(17)\end{array}$ & & $\begin{array}{l}83 \\
(6)\end{array}$ & & $\begin{array}{l}-37 \\
-37 \\
-30 \\
-21 \\
-16\end{array}$ & 58.1 & $\begin{array}{l}58.1 \\
57.4 \\
57.3 \\
57.2\end{array}$ & $\begin{array}{l}0.0 \\
-0.7 \\
-0.8 \\
-0.9\end{array}$ & $\begin{array}{l}2288 \\
2366\end{array}$ & $\begin{array}{l}39.4 \\
41.4\end{array}$ & 22 & -0.9 & +78 & $\begin{array}{l}1480 \\
1605\end{array}$ & $\begin{array}{l}25.8 \\
28.0\end{array}$ & 9 & -0.1 & +125 \\
\hline & $\begin{array}{l}146 \\
\text { (11) } \\
165 \\
\text { (12) }\end{array}$ & $\begin{array}{l}<.01 \\
<.01\end{array}$ & $\begin{array}{l}76 \\
(7) \\
80 \\
(8)\end{array}$ & $\begin{array}{l}<.05 \\
<.4>.3\end{array}$ & $\begin{array}{r}+10 \\
+19 \\
+31 \\
+38 \\
+43 \\
+75 \\
+78 \\
\square \\
\square-18 \\
-20\end{array}$ & & $\begin{array}{l}53.3 \\
53.2 \\
53.2 \\
53.1 \\
52.8 \\
53.6 \\
53.6 \\
54.6 \\
54.5\end{array}$ & $\begin{array}{l}-4.8 \\
-4.9 \\
-4.9 \\
-5.0 \\
-5.3 \\
-4.6 \\
-4.5 \\
-3.5 \\
-3.6\end{array}$ & $\begin{array}{l}1977 \\
2193 \\
1957 \\
2287\end{array}$ & $\begin{array}{l}37.1 \\
41.5 \\
36.5 \\
41.9\end{array}$ & $\begin{array}{l}36 \\
24 \\
32 \\
\\
22\end{array}$ & $\begin{array}{r}+4.0 \\
-0.4 \\
+0.8 \\
+1.0\end{array}$ & $\begin{array}{l}-389 \\
+216 \\
-236 \\
+330\end{array}$ & $\begin{array}{l}1496 \\
1539 \\
1642 \\
1433 \\
1439\end{array}$ & $\begin{array}{l}26.1 \\
26.9 \\
30.9 \\
26.7 \\
26.4\end{array}$ & $\begin{array}{l}31 \\
21 \\
7 \\
40 \\
22\end{array}$ & $\begin{array}{c}+4.0 \\
-0.1 \\
-0.1 \\
+0.5 \\
+0.9\end{array}$ & \begin{tabular}{|l}
-109 \\
+43 \\
+103 \\
-209 \\
+6
\end{tabular} \\
\hline \multirow{2}{*}{$\begin{array}{c}\stackrel{8}{8} \\
\text { \$69 } \\
\text { (\$5504) }\end{array}$} & $\begin{array}{l}201 \\
(14)\end{array}$ & & (11) & & $\begin{array}{l}-42 \\
-19 \\
-16 \\
-12 \\
-9\end{array}$ & 62.2 & $\begin{array}{l}62.60 \\
64.9 \\
65.3 \\
65.4\end{array}$ & $\begin{array}{l}+0.4 \\
+2.7 \\
+3.1 \\
+3.2\end{array}$ & $\begin{array}{l}2331 \\
2332\end{array}$ & $\begin{array}{l}37.2 \\
35.9\end{array}$ & 3 & +2.3 & +1 & $\begin{array}{l}1833 \\
1880\end{array}$ & $\begin{array}{l}28.1 \\
28.4\end{array}$ & 3 & +0.1 & th \\
\hline & $\begin{array}{l}178 \\
\text { (11) } \\
\end{array}$ & $k .01$ & $\begin{array}{l}89 \\
(9) \\
\end{array}$ & $\langle<.2\rangle .1$ & $\begin{array}{r}+30 \\
+377 \\
+40 \\
+44 \\
+46 \\
+53 \\
\end{array}$ & & $\begin{array}{l}65.6 \\
65.8 \\
65.7 \\
66.2 \\
65.9 \\
65.9\end{array}$ & $\begin{array}{r}+3.4 \\
+3.6 \\
+3.5 \\
+4.0 \\
+3.7 \\
+3.7 \\
\end{array}$ & \begin{tabular}{|l|}
2184 \\
2232 \\
2187
\end{tabular} & $\begin{array}{l}33.3 \\
33.9 \\
33.3\end{array}$ & $\begin{array}{l}46 \\
10 \\
6\end{array}$ & $\begin{array}{l}+0.7 \\
+0.1 \\
+0.2\end{array}$ & $\begin{array}{l}-148 \\
+48 \\
-45\end{array}$ & $\begin{array}{l}2023 \\
1905 \\
1812 \\
\end{array}$ & $\begin{array}{l}30.8 \\
26.8 \\
27.5 \\
\end{array}$ & $\begin{array}{l}46 \\
7 \\
9 \\
\end{array}$ & $\begin{array}{r}+0.4 \\
+0.4 \\
-0.3\end{array}$ & $\begin{array}{l}+163 \\
-118 \\
-93\end{array}$ \\
\hline \multirow[t]{2}{*}{$8_{42}^{2}+2$} & $\begin{array}{l}172 \\
\text { (18) }\end{array}$ & & (4) & & $\begin{array}{l}-42 \\
-19 \\
-16 \\
-12 \\
-9\end{array}$ & 73.1 & $\begin{array}{l}72.8 \\
73.8 \\
72.8 \\
73.8\end{array}$ & $\begin{array}{l}-0.3 \\
+0.7 \\
-0.3 \\
0.7\end{array}$ & $\begin{array}{l}3002 \\
3023\end{array}$ & $\begin{array}{l}41.2 \\
41.0\end{array}$ & 3 & +1.0 & +21 & $\begin{array}{l}3057 \\
3047\end{array}$ & $\begin{array}{l}42.0 \\
41.3\end{array}$ & 3 & +1.0 & $-10^{\circ}$ \\
\hline & $\begin{array}{l}162 \\
(15)\end{array}$ & $\langle .2\rangle .1$ & $\begin{array}{l}83 \\
\text { (11) }\end{array}$ & k.01 & $\begin{array}{r}+30 \\
+37 \\
+40 \\
+44 \\
+46 \\
+53\end{array}$ & & $\begin{array}{l}72.3 \\
72.0 \\
71.7 \\
71.7 \\
60.6 \\
71.6\end{array}$ & $\begin{array}{l}-0.8 \\
-1.1 \\
-1.4 \\
-1.4 \\
-2.5 \\
-1.5\end{array}$ & \begin{tabular}{|l|}
2639 \\
2708 \\
2657
\end{tabular} & $\begin{array}{l}36.5 \\
37.8 \\
37.6\end{array}$ & $\begin{array}{l}46 \\
10 \\
3\end{array}$ & \begin{tabular}{|l|}
-1.5 \\
-0.6 \\
-1.1
\end{tabular} & $\begin{array}{l}-384 \\
+69 \\
-51\end{array}$ & $\begin{array}{l}3259^{\circ} \\
3178^{\circ} \\
3063\end{array}$ & $\begin{array}{l}45.3 \\
44.3 \\
42.8\end{array}$ & $\begin{array}{l}46 \\
7 \\
9\end{array}$ & $\begin{array}{c}-1.8 \\
-0.3 \\
-0.1\end{array}$ & $\mid \begin{array}{l}+212 \\
-81 \\
-115\end{array}$ \\
\hline
\end{tabular}

tive error, and $\sigma_{1}, \sigma_{2} \ldots$ etc. denote individual analytical errors. Using this formula, the average standard deviation of the entire procedure was .014, equivalent to about $40 \mathrm{mEq}$. for a $70-\mathrm{Kg}$. adult. This calculated value subsequently proved to be in substantial agreement with that found in 29 consecutive determinations on six patients in which the mean difference of duplicate values for TENa obtained at equilibrium, namely 24 and 26 hours, was 40.4 $\mathrm{mEq}$, uncorrected for weight. The value for TEK was based on primary data similar to those used for sodium, but the standard deviation of the counts among the three urine samples has also been included, the value amounting to 3.8 per cent. Based on average rates of $10,000,1440$ and $220 \mathrm{cpm}$ for infusion fluid, urine samples, and background total counts of $20,00012,000$ and 30,000 , respectively, the coefficients of variance are $.0092, .0070$ and
.0058. A value of .01 has been given to the chemical analysis for $\mathrm{K}$ on the basis of analytical data similar to those obtained for sodium. The cumulative error of these five values becomes .041 , equivalent to about $80 \mathrm{mEq}$. for a $60-\mathrm{Kg}$. female (12) and $130 \mathrm{mEq}$. for a $70-\mathrm{Kg}$. male (3). Standard deviations of blood pressures were calculated from the mean of readings obtained during the final 10 days of both the high (control) and low (test) sodium phases. Fisher's " $t$ " test was used as a criterion of significance between the mean values so found, with $\mathrm{p}=$ $<.01$.

\section{OBSERVATIONS}

The results of these studies are summarized in Tables Ia, Ib, and Ic in which the data are listed and divided among those subjects with a fall in 


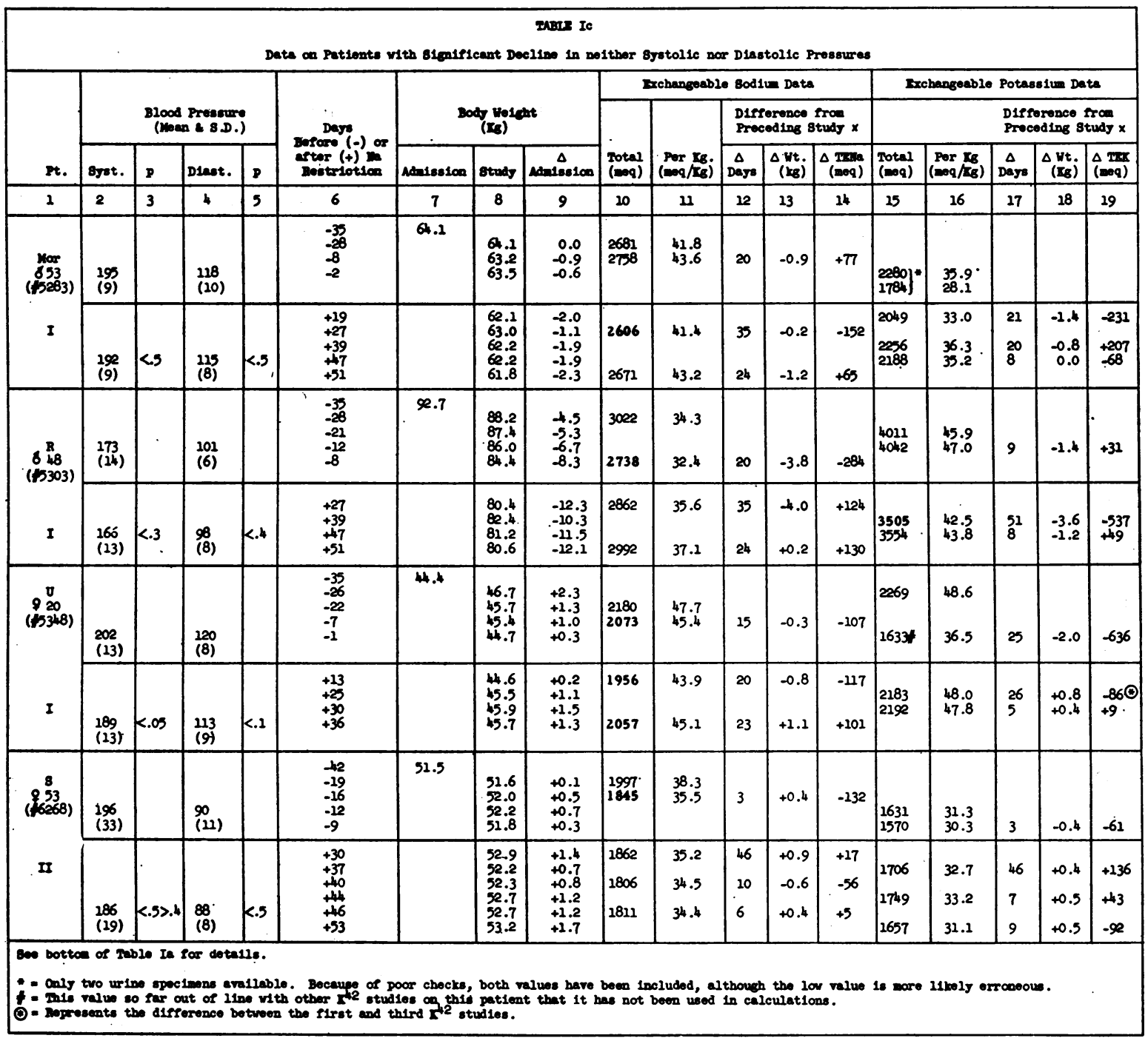

both, either, or neither systolic and diastolic pressures, respectively. Statistically significant declines in both systolic and diastolic pressures were shown by 3 of the 11 patients following this degree of sodium restriction, a fraction that approximates our experience with a similar diet during the past six years, as well as the experience of others (1315). Eight of these patients had a decrease in TENa of 3 S.D. or more during the low $\mathrm{Na}$ phase and were divided without relation to the effects on blood pressure. This was noted earlier (1).

The values obtained for TEK on all patients were in agreement with those reported for normal males (3) and females (12). Among the 11 patients limitation of $\mathrm{Na}$ was followed by a statistically significant change in TEK in only two sub- jects, both males: Mon (Table Ia) and $\mathrm{R}$ (Table Ic). In the first instance there was an increase of $469 \mathrm{mEq}$. in TEK in association with a decline in blood pressure, and in the second there was a fall in TEK of $537 \mathrm{mEq}$. with no change in blood pressure. The remaining nine patients showed variable and modest responses in TEK which differed somewhat between the two groups. TEK in Group I was found to vary in minor degree about the control values, except for patient $R$ as noted. By contrast, a fairly uniform reaction occurred in the members of Group II : 37 days after $\mathrm{Na}$ restriction TEK was elevated over control levels in all five patients. The increase exceeded 3 S.D. in only one patient (Mon, Table Ia) on a weight and sex basis, but the increases were all 


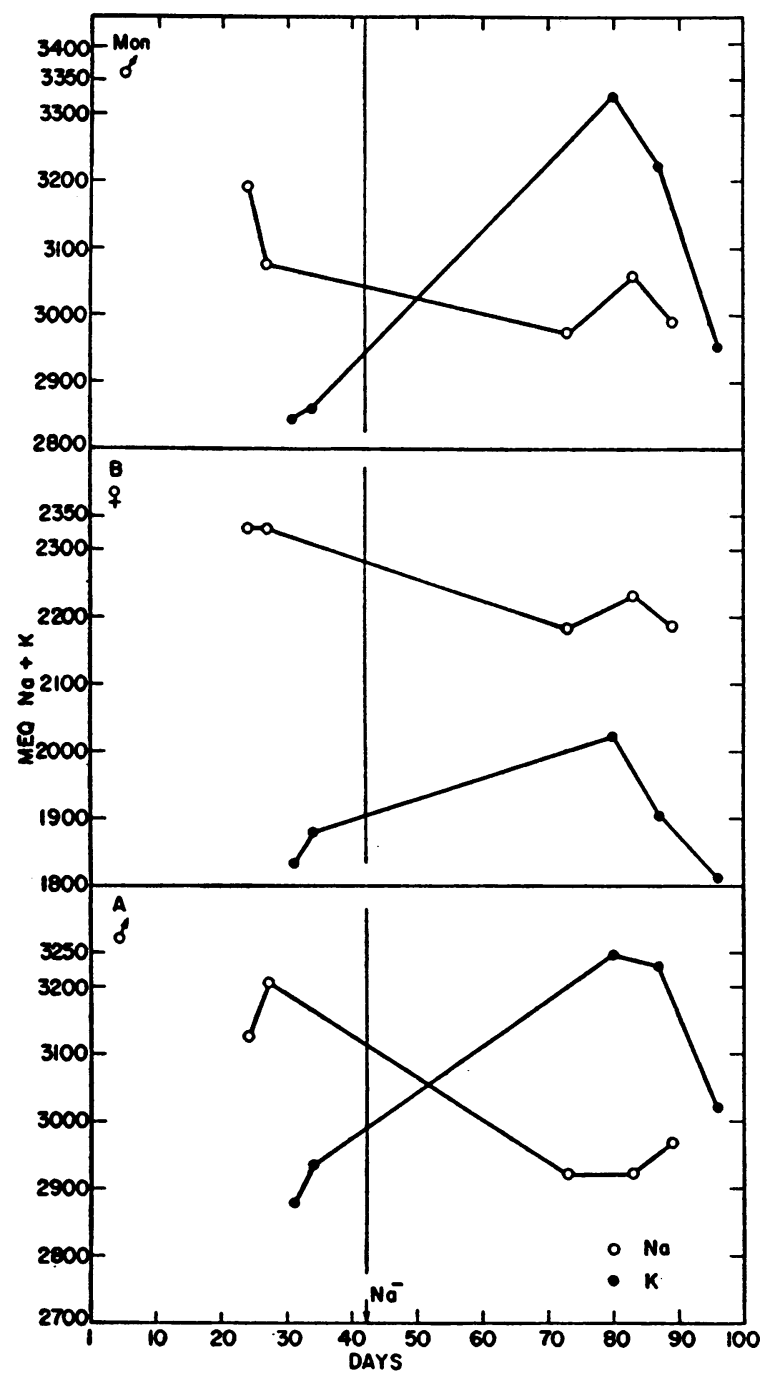

Fig. 1. TENa ANd TEK before ANd AFter Na Restriction in Three Patients

The vertical line on abscissa indicates day on which added $\mathrm{Na}$ (180 mEq.) was omitted from diet.

similar in magnitude ( +136 to $+469 \mathrm{mEq}$.). Recheck of TEK 44 and again 53 days after $\mathrm{Na}$ restriction revealed that the initial elevation was not permanent for by the 53rd day, the data obtained were in substantial agreement with those found during the control period. In Figure 1, the values on several representative patients of Group II have been plotted against time from which it will be seen that the decline in TEK was gradual. This suggested that the initial elevation noted after $\mathrm{Na}$ restriction represented a physiologic response rather than a methodological aberration. Furthermore, from the characteristic urinary patterns shown in Figure 2, it may be seen that $\mathrm{Na}$ limitation had no apparent effect on $\mathrm{K}$ excretion. This confirms earlier observations (1). Repeated measurements were made of serum $\mathrm{Na}$ and $\mathrm{K}$ throughout the course of the study and showed no significant deviations during either phase of the regimen.

\section{INTERPRETATION}

In confirmation of earlier work (1), these studies indicate that sodium restriction does not act by simply "salting out" the organism since desalting, at least as measured by TENa, bore no relationship to the therapeutic effect in these patients. In view of the evidence (16) that in some patients TENa can decrease or increase unrelated to net balance of sodium, the possibility must be considered that TENa may not accurately mirror any desalting which does occur. However, calculation of the net sodium balance in this group by measurement of total urinary sodium assuming a daily stool output of $1.5 \mathrm{mEq}$. per day (1) and an intake of $6 \mathrm{mEq}$. per day, failed to show that in those with the greatest net loss, the most significant declines in blood pressure occurred.

It had been found earlier (1) in four patients subjected to a simliar regimen that loss of exchangeable sodium uniformly exceeded the weight loss which should have occurred if the sodium came from extracellular fluid (ECF). Therefore, the hypothesis was proposed that failure to lose an equivalent amount of weight along with the sodium was due to a movement of water from an extracellular to an intracellular position. Analysis of the data from these 11 patients relative to this point is shown in detail in the accompanying table

Fig. 2. Urinary Excretion of Na and K before and after Na Restriction

The second vertical line on patient $\mathrm{P}$ indicates point at which $\mathrm{NaCl}$ again was added to diet; this patient also received a total of $400 \mathrm{mgm}$. Cortisone, I.M., on 15 and $16 \mathrm{July}$, in association with studies not pertinent to the present paper. The studies on the group as a whole had been completed at this time; the effects on $\mathrm{Na}$ and $\mathrm{K}$ excretion are readily visible. Note that ordinates are logarithmic. 

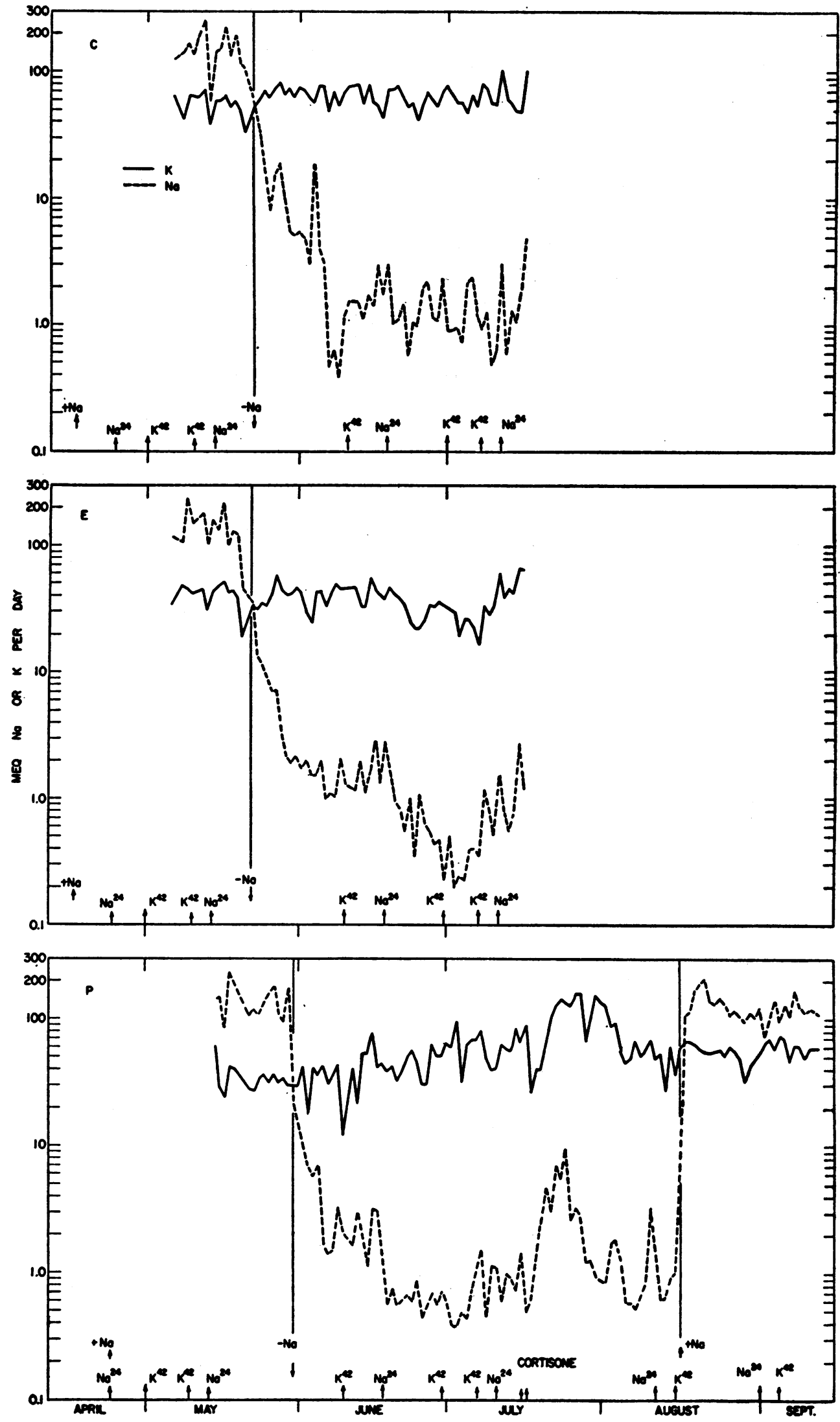

FIG. 2. 
TABLE II

Comparison of determined TENa difference after $\mathrm{Na}$ restriction and theoretical $\mathrm{Na}$ equivalent of the weight change, assuming that all weight changes were due to variations in ECF associated with withdrawal or addition $(P)$ of $\mathrm{Na}$ *

\begin{tabular}{|c|c|c|c|c|c|}
\hline & $\begin{array}{c}\text { Patient } \\
\text { and } \\
\text { No. }\end{array}$ & $\begin{array}{c}\Delta \\
\mathbf{w} t . \\
\left(\boldsymbol{K}_{\mathbf{g}}\right)\end{array}$ & $\begin{array}{c}\text { E.C.F. } \\
\text { equiv. } \\
(\mathrm{A}) \\
(\mathrm{Kg} . \times 140)\end{array}$ & $\underset{\text { (B) }}{\mathbf{T E N a}}$ & $\mathbf{B}-\mathbf{A}$ \\
\hline Group I & $\begin{array}{r}\text { P } 5424 \\
\text { U } 5348 \\
\text { C } 5420 \\
\text { E } 5274 \\
\text { M } 5283 \\
\text { R } 5303\end{array}$ & $\begin{array}{l}-4.0 \\
+1.0 \\
-0.8 \\
-1.0 \\
-2.0 \\
-0.2 \\
-4.0\end{array}$ & $\begin{array}{l}-560 \\
+140 \\
-112 \\
-140 \\
-280 \\
-28 \\
-560\end{array}$ & $\begin{array}{l}-389 \\
+330 \\
-117 \\
-123 \\
-178 \\
-152 \\
+124\end{array}$ & $\begin{array}{r}+171 \\
+190 \\
-\quad 5 \\
+\quad 17 \\
+102 \\
-124 \\
+684\end{array}$ \\
\hline Group II & $\begin{array}{r}\text { A } 5455 \\
\text { B } 5504 \\
\text { L } 5458 \\
\text { Mon } 5472 \\
\text { S } 5268\end{array}$ & $\begin{array}{l}-1.2 \\
+0.7 \\
-1.5 \\
+0.3 \\
+0.9\end{array}$ & $\begin{array}{l}-168 \\
+98 \\
-210 \\
+\quad 42 \\
+126\end{array}$ & $\begin{array}{l}-285 \\
-148 \\
-384 \\
-101 \\
+17\end{array}$ & $\begin{array}{l}-117 \\
-246 \\
-174 \\
-143 \\
-109\end{array}$ \\
\hline
\end{tabular}

* These values were obtained from the last TENa determination before, and the first TENa after, restriction of $\mathrm{Na}$ in the diet. The second value for patient $\mathrm{P}$ represents the difference between the last TENa before and the first TENa after, the re-addition of $\mathrm{Na}$ in the diet.

(Table II) which includes changes in weight and TENa recorded between the last measurement of $\mathrm{TENa}$ before $\mathrm{Na}$ restriction and the first such measurement after restriction (patient $P$ includes a period of sodium restriction followed by one of sodium addition).

The present data neither wholly confirm nor refute the hypothesis proposed above. It will be seen that the five patients in Group II are in agreement with the proposed interpretation in that weight loss fell short of equivalence with the decrease in TENa. But in Group I only patient M followed the same pattern although $P$, upon readding $\mathrm{Na}$, gained less weight than would have been predicted from her increase in TENa, thereby suggesting that if the increase in TENa was associated with an increase in ECF, the necessary fluid came from intracellular water. The other five patients in this group were not in agreement with the hypothesis suggested. In two, decline in TENa and weight were virtually equivalent while the remaining three showed declines in TENa of less than the weight equivalence by 102 to $684 \mathrm{mEq}$. $\mathrm{Na}$. It is of interest that the two groups differed by such an extent and it is not simply explained by the effects of weight loss with the relative absence of such loss in Group II versus the considerable loss in some members of Group I : patients $U$ and
C in Group I lost insignificant amounts of weight, yet failed to lose more than an equivalent amount of sodium measured by TENa. Two of the five patients in Group II lost slightly greater amounts of weight than did $U$ and $C$, yet had an associated decline in TENa of significantly greater equivalence. Parenthetically, a normal male that underwent the regimen in Group II, and who forms the subject of a separate report (16) behaved like the members of Group I rather than those in his own group; this suggested that changes in environmental temperature, diet, or unknown technical factors could not be called in to account for the difference between the groups. It seems at least as reasonable to interpret some of the changes in TENa as being fluctuations in the amount of body $\mathrm{Na}$ available for exchange with the isotope, presumably due to that in the reservoir contained in bone (17). Sjögren (18) made a similar suggestion after treating a patient with rheumatoid arthritis with $20 \mathrm{mg}$. DCA for 60 days and observing

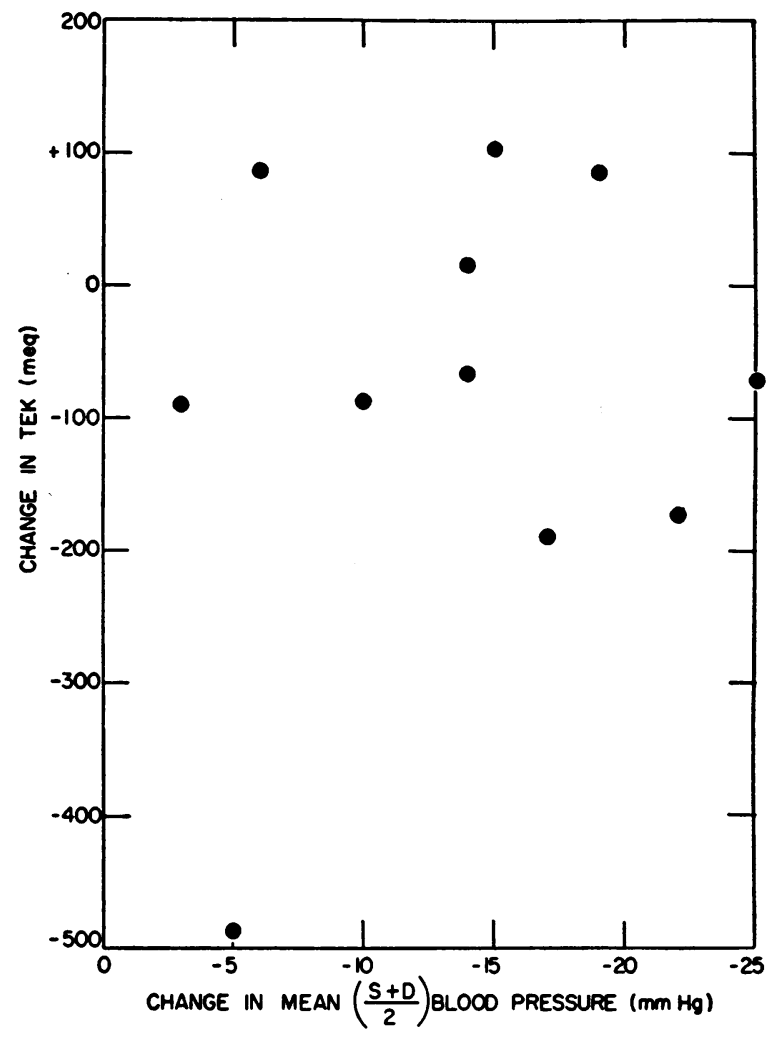

Fig. 3. Lack of Correlation between Changes $\frac{\text { (Systolic + Diastolic) }}{2}$

Blood Pressure and

\section{Change in TEK}


TABLE III

Summary of changes in blood pressure, TENa and TEK

\begin{tabular}{ccccc}
\hline \hline & $\begin{array}{c}\text { Significant* } \\
\text { decrease in B.P. }\end{array}$ & $\begin{array}{c}\text { Significant } \\
\text { decrease } \\
\text { in TENa }\end{array}$ & $\begin{array}{c}\text { Significant } \\
\text { change } \\
(+ \text { or } \\
\text { in TEK }\end{array}$ \\
\cline { 2 - 5 } Patient & Syst. & Diast. & + & 0 \\
$\mathbf{C}$ & + & + & + & 0 \\
A & + & + & + & + \\
Mon & + & + & 0 & 0 \\
E & + & 0 & + & 0 \\
$\mathbf{P}$ & + & 0 & + & 0 \\
B & + & 0 & + & 0 \\
L & 0 & + & + & 0 \\
Mor & 0 & 0 & + & 0 \\
$\mathbf{R}$ & 0 & 0 & 0 & 0 \\
$\mathbf{U}$ & 0 & 0 & + & 0 \\
S & 0 & 0 & & 0
\end{tabular}

* Statistically significant.

an increase of $700 \mathrm{mEq}$. in TENa in the presence of a net $\mathrm{Na}$ loss of $330 \mathrm{mEq}$.

The data do not suggest that drastic limitation of $\mathrm{Na}$ intake acts by effects on $\mathrm{K}$, at least as measured by TEK. The frank lack of correlation between blood pressure changes and TEK is shown in Figure 3. Further, as indicated in Table III, there seemed to be no relationship between changes in TEK, and those in TENa. The types of measurements used are, of course, relatively gross and it is possible that while no constant effect on $K$ metabolism was found, fine changes were effected by the regimen but were obscured by the daily variations in $\mathrm{K}$ intake and the magnitude of the $\mathrm{K}$ pool. No explanation is presently available for the transient rise of TEK found in Group II and not in Group I. With $\mathrm{Na}$ restriction, it is known that $\mathrm{K}$ largely replaces $\mathrm{Na}$ as the dominant ion in sweat (5). It is therefore possible that the warm weather associated with the last half of the period in which Group I was studied could have led to $K$ loss of considerable degree with resulting reflection upon TEK; and it is true that in this group as a whole, TEK declined slightly over control values.

The weight changes failed to account for variations in TENa as mentioned earlier; this was also true for TEK as indicated by data in columns 18 and 19 , Tables $\mathrm{Ia}, \mathrm{Ib}$, and Ic. It is reasonable to suggest that here, too, the variability found is accounted for by changes due to the bone reservoir of $\mathrm{K}(17)$.

\section{SUMMARY}

1. A series of 11 patients with essential hypertension has been studied before and after restriction of $\mathrm{Na}$ intake to approximately $6 \mathrm{mEq}$. per day.
2. After restriction of $\mathrm{Na}$ intake, no correlations were found between changes in blood pressure and either total exchangeabse sodium or potassium.

3. It was suggested that some of the variations which occurred in TENa and TEK were due to fluctuations in the amount of body sodium and potassium made available for exchange with the isotopes, presumably due to the reservoir of these elements contained in bone.

\section{ACKNOWLEDGMENTS}

We wish to thank Miss Ruth Sovocool, Mr. John Ruscica, and Mrs. Miriam Brown for their invaluable technical assistance, and the Misses Susan Lamb and Norma Gillespie, dietitians, for supervision of the diets. The fine cooperation of the nurses, particularly Miss Josephine O'Connell, R.N. and Miss Rosemarie McCann, R.N., is acknowledged.

\section{REFERENCES}

1. Dole, V. P., Dahl, L. K., Cotzias, G. C., Dziewiatkowski, D. D., and Harris, C., Dietary treatment of hypertension. II. Sodium depletion as related to the therapeutic effect. J. Clin. Invest., 1951, 30, 584.

2. Edelman, I. S., James, A. H., Baden, H., and Moore, F. D., Electrolyte composition of bone and the penetration of radiosodium and deuterium oxide into dog and human bone. J. Clin. Invest., 1954, 33, 122.

3. Corsa, L., Jr., Olney, J. M., Jr., Steenburg, R. W., Ball, M. R., and Moore, F. D., The measurement of exchangeable potassium in man by isotope dilution. J. Clin. Invest., 1950, 29, 1280.

4. Kempner, W., Treatment of hypertensive vascular disease with rice diet. Am. J. Med., 1948, 4, 545.

5. Dole, V. P., Dahl, L. K., Cotzias, G. C., Eder, H. A., and Krebs, M. E., Dietary treatment of hypertension. Clinical and metabolic studies of patients on the rice-fruit diet. J. Clin. Invest., 1950, 29, 1189.

6. Watkin, D. M., Froeb, H. F., Hatch, F. T., and Gutman, A. B., Effects of diet in essential hypertension. Am. J. Med., 1950, 9, 428.

7. Dole, V. P., Dahl, L. K., Schwartz, I. L., Cotzias, G. C., Thaysen, J. H., and Harris, C., Dietary treatment of hypertension. III. The effect of protein on appetite and weight. J. Clin. Invest., 1953, 32, 185.

8. Peters, J. P., and Van Slyke, D. D., Quantitative Clinical Chemistry. Vol. I, Interpretations. Baltimore, Williams \& Wilkins, 1932, p. 766.

9. Bordley, J., III, Connor, C. A. R., Hamilton, W. F., Kerr, W. J., and Wiggers, C. J., Recommendations for human blood pressure determinations by sphygmomanometers. Circulation, 1951, 4, 503. 
10. Jarvett, A. A., Statistical methods used in the measurement of radioactivity. U. S. Atomic Energy Commission $\wedge$ AECU-262, Mon P-126, 17 June, 1946.

11. Fisher, R. A., Statistical methods for research workers. 9th Revised Edition, London, Oliver and Boyd, 1944, p. 116.

12. Aikawa, J. K., Harrell, G. T., and Eisenberg, B., The exchangeable potassium content of normal woman. J. Clin. Invest., 1952, 31, 367.

13. Grollman, A., Harrison, T. R., Mason, M. F., Baxter, J., Crampton, J., and Reichsman, F., Sodium restriction in the diet for hypertension. J. A. M. A., 1945, 129, 533.
14. Schroeder, H. A., Goldman, M. L., Futcher, P. H., and Hunter, M., Low sodium chloride diets in hypertension. J. A. M. A., 1949, 140, 458.

15. Corcoran, A. C., Taylor, R. D., and Page, I. H., Controlled observations on the effect of low sodium dietotherapy in essential hypertension. Circulation, 1951, 3, 1.

16. Dahl, L. K., Stall, B. G., and Cotzias, G. C., To be published.

17. Bergstrom, W. H., Bone as a sodium and potassium reservoir. J. Clin. Invest., 1952, 31, 617.

18. Sjögren, B., Changes in total body sodium and potassium during prolonged administration of cortisone and desoxycortisone acetate. Acta Endocrinol., 1952, 10, 40. 